\title{
Correction to: graph analysis of structural brain networks in Alzheimer's disease: beyond small world properties
}

\author{
Majnu John ${ }^{1,2,3} \cdot$ Toshikazu Ikuta $^{4} \cdot$ Janina Ferbinteanu $u^{5,6}$
}

Published online: 26 September 2020

๑) Springer-Verlag GmbH Germany, part of Springer Nature 2020

\section{Correction to: Brain Struct Funct (2017) 222:923-942 https://doi.org/10.1007/s00429-016-1255-4}

The original version of the article contained an error in the electronic supplementary material.

The caption of the figure in the electronic supplementary material was omitted.

The caption should read as

SUPPLEMENTARY FIGURE 1: sparsity levels differ in AD patients and control groups for the same correlational thresholds. Data of AD group are shown in red, data of control group are in blue. The two curves were obtained based on AD patient and control data, respectively, by computing sparsity (proportion of edges out of the total number of possible edges) of graphs constructed by using as

The original article can be found online at https://doi.org/10.1007/ s00429-016-1255-4.

Electronic supplementary material The online version of this article (https://doi.org/10.1007/s00429-020-02140-4) contains supplementary material, which is available to authorized users.

Janina Ferbinteanu

janina.ferbinteanu@downstate.edu; ferbinteanu@gmail.com

1 The Feinstein Institute for Medical Research, North ShoreLIJ Health System, Manhasset, NY, USA

2 The Zucker Hillside Hospital, Psychiatry Research, North Shore-LIJ Health System, Glen Oaks, NY, USA

3 Department of Mathematics, Hofstra University, Hempstead, NY, USA

4 Department of Communication Sciences and Disorders, University of Mississippi, Oxford, USA

5 Department of Physiology and Pharmacology, SUNY Health Science Center at Brooklyn, Brooklyn, NY 11203, USA

6 Department of Neurology, SUNY Health Science Center at Brooklyn, Brooklyn, NY, USA correlation threshold all correlation values between 0 and 1 in steps of 0.001. One sparsity level corresponds to two different correlation coefficients for patient and control groups, and conversely, one correlation coefficient value corresponds to two different sparsity levels (dashed lines). Sparsity level of $5 \%$ corresponded to a correlational threshold of 0.5715 in patients and 0.5225 in controls; sparsity level of $34 \%$ corresponded to a correlational threshold of 0.3765 in patients and 0.3515 in controls.

Publisher's Note Springer Nature remains neutral with regard to jurisdictional claims in published maps and institutional affiliations. 